\title{
Analysis of the role of shear waves in transcranial photoacoustic tomography with a planar geometry
}

Robert W. Schoonover, Lihong V. Wang, Mark A. Anastasio

Robert W. Schoonover, Lihong V. Wang, Mark A. Anastasio, "Analysis of the role of shear waves in transcranial photoacoustic tomography with a planar geometry," Proc. SPIE 8223, Photons Plus Ultrasound: Imaging and Sensing 2012, 822320 (23 February 2012); doi: 10.1117/12.907389

SPIE. Event: SPIE BiOS, 2012, San Francisco, California, United States 


\title{
Analysis of the role of shear waves in transcranial photoacoustic tomography with a planar geometry
}

\author{
Robert W. Schoonover, Lihong V. Wang, and Mark A. Anastasio \\ Department of Biomedical Engineering, \\ Washington University in St. Louis, \\ St. Louis, MO, USA
}

\begin{abstract}
We report on an investigation of the role of shear waves in transcranial PAT brain imaging. Using a recently developed PAT image reconstruction method for use with layered media, we quantify the extent to which accounting for shear waves in the reconstruction method can improve image quality. The effects of shear waves propagating in the solid layer on the ability to estimate Fourier components of the object are investigated as a function of the thickness of the layer supporting shear waves as well as the incidence angle of the field in the planewave representation. These results clarify the role of shear waves in transcranial PAT image formation and indicate that further research is warranted to develop reconstruction algorithms that account for shear waves.
\end{abstract}

Keywords: photoacoustic tomography, image reconstruction, shear waves

\section{INTRODUCTION}

Photoacoustic Tomography (PAT) is a biomedical imaging technique, based on the photoacoustic effect, which combines the optical absorption contrast of tissue with the high spatial resolution of ultrasound imaging techniques. ${ }^{1-4}$ In PAT, the object of interest is illuminated with a short optical pulse and an acoustic pressure signal is generated via the photoacoustic effect. ${ }^{2,3}$ The pressure signal is subsequently measured by wide-band ultrasonic transducers at a number of locations outside the object and the total absorbed optical energy density is estimated from the measured data via numerical image reconstruction algorithms. The utility of PAT has been demonstrated in a number of in vivo studies of biological structure and function ${ }^{5-7}$ and in facilitating a number of medically-relevant diagnostic tasks. ${ }^{2,8-13}$

Functional imaging of mouse brains has been successfully demonstrated with PAT. ${ }^{5,6}$ However, only qualitatively useful images of brain structures have been demonstrated for larger animals. ${ }^{14-17}$ For larger animals, including primates, the measured photoacoustic signal becomes distorted upon passage through the skull bone(s) through the processes of dispersion, absorption, strong reflections, and longitudinal-to-shear mode conversion. This distortion can result in degraded spatial resolution or the presence of artifacts in the reconstructed images. Time-reversal algorithms for PAT have been shown to compensate for density variations, dispersion and absorption, ${ }^{18}$ but they do not currently account for media that support shear wave propagation.

When the to-be-imaged object is embedded in a planar, layered medium, the effects of absorption, dispersion, and shear-mode conversion can be properly compensated for by use of a recently derived PAT reconstruction formula. ${ }^{19}$ The reconstruction formula estimates the Fourier components of the absorbed optical energy density using an angular spectrum representation of the photoacoustic field. In this work, the PAT reconstruction formula valid for planar, layered media is employed to evaluate the role of modeling shear waves in PAT. Specifically, the transmission function for a three layer system when shear waves are modeled is compared to the transmission function for a three layer system for a system that does not compensate for shear waves. Comparisons are made based on the incident angle of the field in the plane wave representation as well as on the thickness of the solid layer.*

Further author information: (Send correspondence to RWS)

RWS: E-mail: rschoono@seas.wustl.edu,anastasio@wustl.edu

${ }^{*}$ This proceeding is based on R.W. Schoonover, L.V. Wang, and M.A. Anastasio, "A numerical investigation of the effects of shear waves in transcranial photoacoustic tomography with a planar geometry," under review, J. Biomed. Opt. (2012).

Photons Plus Ultrasound: Imaging and Sensing 2012, edited by Alexander A. Oraevsky, Lihong V. Wang, Proc. of SPIE Vol. 8223, 822320 - (c) 2012 SPIE · CCC code: 1605-7422/12/\$18 - doi: 10.1117/12.907389 


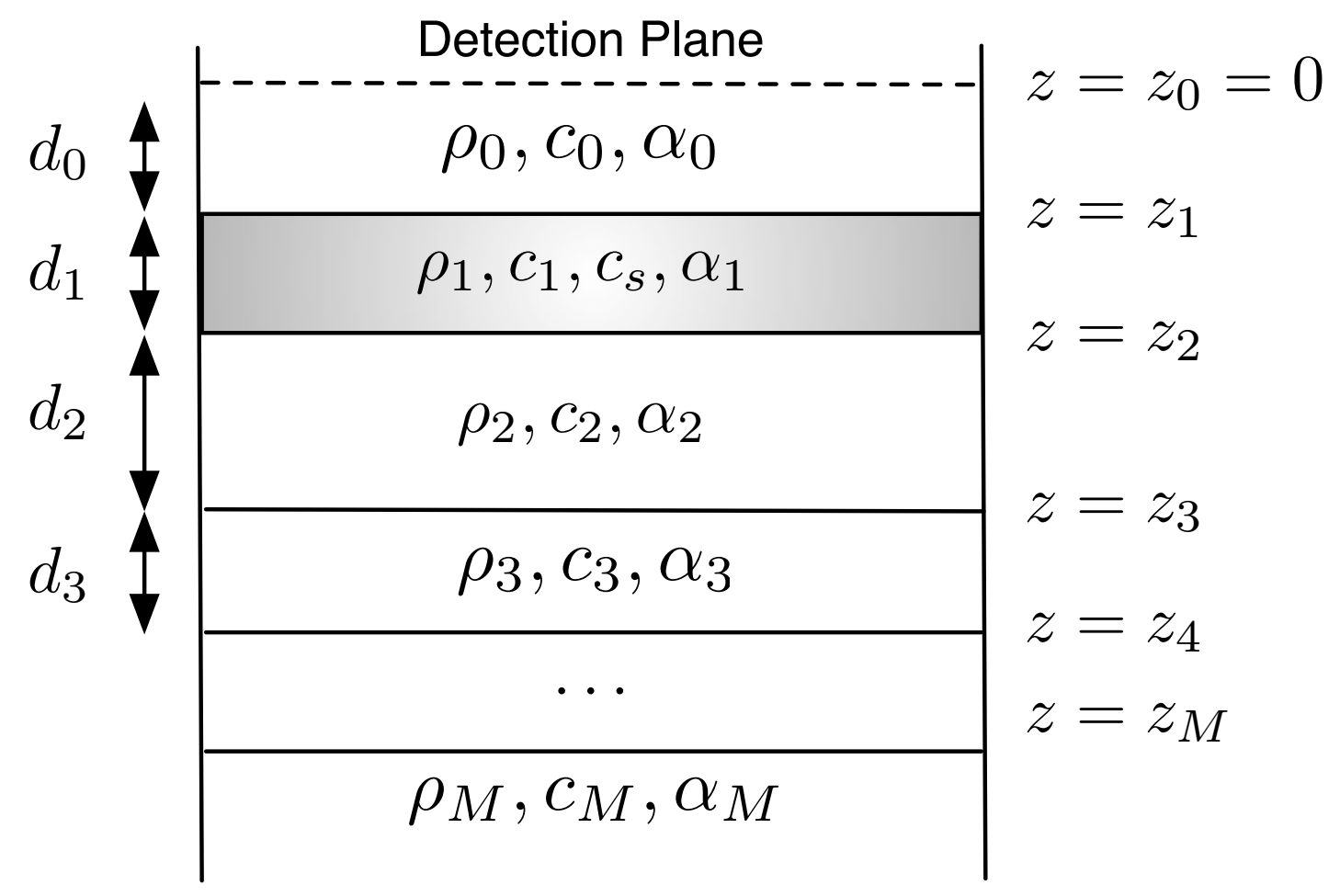

Figure 1. A diagram of the layered system. Ultrasonic detection occurs in the plane $z=0$. Layers 2 and 0 are assumed to be fluids, with densities $\rho_{m}$ and speed of sound $c_{m}$. Layer 0 has thickness $d_{0}$ and the speed of sound and density are consistent with skin tissue. Layer 1 is assumed to be a solid, bone, with density $\rho_{1}$, longitudinal speed of sound $c_{l}$, shear speed of sound $c_{s}$, thickness $d_{1}$, and longitudinal and shear absorption coefficients $\bar{\alpha}_{l}$ and $\bar{\alpha}_{s}$. The dispersive speeds of sound are referenced to $1 \mathrm{MHz}$. Layer 2 is assumed to be unbounded in the negative $z$-direction, the speed of sound and density are consistent with tissue.

\section{BACKGROUND}

The geometry of the layered system is shown in Fig. 1. The to-be-imaged object is contained in layer 2, characterized by speed of sound $c_{2}$ and density $\rho_{2}$, where both are consistent with biological tissue (e.g. a fluid). The photoacoustic signal is detected in layer 0 , on the plane $z=0$, characterized by thickness $d_{0}$, speed of sound $c_{0}$, and density $\rho_{0}$. Layer 1 is assumed to be a solid (e.g., skull bone) of thickness $d_{1}$, which supports shear wave propagation. The solid is assumed to be dispersive and absorptive for both longitudinal and shear waves.

In photoacoustic tomography, the measured acoustic pressure is used employed to estimate the absorbed optical energy density, denoted by $A(\mathbf{r})$. The space-frequency domain representation, $\tilde{p}(\mathbf{r}, \omega)$, of the acoustic field is related to the space-time representation, $p(\mathbf{r}, t)$, by a Fourier transform, viz.

$$
\tilde{p}(\mathbf{r}, \omega)=\int_{\infty} \mathrm{d} t p(\mathbf{r}, t) e^{i \omega t}
$$

where $\omega$ denotes the temporal frequency, $i \equiv \sqrt{-1}$, and $\mathbf{r}=(x, y, z)$. The pressure field away from the acoustic source can be expressed as

$$
\tilde{p}(\mathbf{r}, \omega)=i \omega \Gamma H(\omega) \iiint_{V} \mathrm{~d}^{3} r^{\prime} G\left(\mathbf{r}, \mathbf{r}^{\prime}, \omega\right) A\left(\mathbf{r}^{\prime}\right),
$$

where $G\left(\mathbf{r}, \mathbf{r}^{\prime}, \omega\right)$ is an appropriate Green function that accounts for the detection system and appropriate boundary conditions, $\Gamma$ is the Grueneisen parameter, and $H(\omega)$ is the temporal Fourier transform of the exciting optical 
pulse's temporal profile. In Cartesian coordinates, $A(\mathbf{r}) \equiv A(x, y, z)$. For planar detection, the Green function has been previously expressed for the cases of a homogeneous medium ${ }^{20}$ and a layered, fluid medium. ${ }^{19,21}$

Let $\bar{p}\left(k_{x}, k_{y}, \omega\right)$ denote the two-dimensional (2D) spatial Fourier transform of the pressure data $\tilde{p}(x, y, z, \omega)$ evaluated on the measurement plane $z=0$ :

$$
\bar{p}\left(k_{x}, k_{y}, \omega\right)=\iint_{\infty} \mathrm{d} x \mathrm{~d} y \tilde{p}(x, y, z=0, \omega) e^{-i\left(k_{x} x+k_{y} y\right)} .
$$

Further, let $\mathcal{A}\left(k_{x}, k_{y}, k_{z}\right)$ denote the 3D Fourier transform of $A(x, y, z)$ :

$$
\mathcal{A}\left(k_{x}, k_{y}, k_{z}\right)=\iiint_{\infty} \mathrm{d} x \mathrm{~d} y \mathrm{~d} z A(x, y, z) e^{-i\left(k_{x} x+k_{y} y+k_{z} z\right)} .
$$

It has been shown ${ }^{19,20}$ that certain Fourier components of $A(\mathbf{r})$ can be estimated from the measured pressure, $p$, via the relation

$$
\mathcal{A}\left(k_{x}, k_{y}, k_{z}^{(2)}\right)=\frac{-2 k_{z}^{(2)}}{\omega \Gamma H(\omega) T\left(\mathbf{k}_{\|}, \omega\right)} \frac{\rho_{2} c_{2}}{\rho_{0} c_{0}} e^{-i k_{z}^{(0)} d_{0}} \bar{p}\left(k_{x}, k_{y}, \omega\right),
$$

where $\mathbf{k}_{\|}=\left(k_{x}, k_{y}, 0\right)^{T}$ and

$$
k_{z}^{(2)}\left(\mathbf{k}_{\|}, \omega\right)=\sqrt{\omega^{2} / c_{2}^{2}-k_{x}^{2}-k_{y}^{2}} .
$$

Here, $d_{0}$ is the distance between the detector and the nearest boundary of the solid layer, and $T\left(\mathbf{k}_{\|}, \omega\right)$ is the planewave amplitude transmission function (ATF). The ATF denotes the relative amplitude and phase of a unit-amplitude planewave, incident on the layered medium from below, traveling in a direction defined by $\mathbf{k}_{\|}$ at temporal frequency $\omega$, and exiting the layered structure at the final layer interface. The estimable values of $\mathcal{A}$ lie inside a sphere in $k$-space of radius $k_{\text {rad }}=\omega_{\max } / c_{2}$, where $\omega_{\max }$ denotes the largest measured temporal frequency. The explicit form for $T$ has been derived previously. ${ }^{19}$ The ATF encapsulates the effects of dispersion, absorption, longitudinal-to-shear wave conversion, and the reflection and transmission of acoustic waves at the interfaces at $z=z_{1}$ and $z=z_{2}$. When the medium is homogeneous, $\left|T\left(\mathbf{k}_{\|}, \omega\right)\right|=1$. This relationship holds for all $\mathbf{k}_{\|}$and $\omega$. For a planar, layered medium, the ATF is a function only of the magnitude of $\mathbf{k}_{\|}$. Without loss of generality, $\mathbf{k}_{\|}$is assumed to be of the form $\left(\omega \sin \theta / c_{2}, 0,0\right)^{T}$ throughout this paper. That is, the wavevector is assumed to lie only in the $x-z$ plane, and is incident on the solid layer at an angle of $\theta$.

An accurate imaging model for transcranial PAT also accounts for dispersion and absorption in the bone. For many tissues of interest, it is known ${ }^{22}$ that the linear absorption coefficient, as a function of temporal frequency, obeys a power law: $\alpha_{m}(\omega)=\bar{\alpha}_{m}|\omega|^{y}$. For $y \neq 1$, the dispersion relationship is given by ${ }^{23}$

$$
\frac{1}{c_{m}(\omega)}=\frac{1}{c_{0 m}}+\alpha_{m} \tan \left(\frac{\pi y}{2}\right)\left(\omega^{y-1}-\omega_{0}^{y-1}\right),
$$

where $c_{m}(\omega)$ is the speed of sound (longitudinal or shear) at frequency $\omega, c_{0 m}$ is the known (measured) speed of sound at reference frequency $\omega_{0}$, and $m=0,1,2$, s. The linear absorption coefficient for longitudinal waves in bone has been experimentally estimated to range from $\alpha_{l}(\omega)=150-500 \mathrm{~Np} / \mathrm{m}$ and the estimated values of $y$ range from 0.8 to $1.3^{22,24-27}$ at ultrasound frequencies.

For the three-layer system assumed for this paper, the bottom layer was assumed to be soft tissue, unbounded in the $-z$ direction, with speed of sound $1483 \mathrm{~m} / \mathrm{s}$ and density $1000 \mathrm{~kg} / \mathrm{m}^{3}$. The middle layer was assumed to be bone (a solid), with longitudinal speed of sound of $2900 \mathrm{~m} / \mathrm{s}$, shear speed $1450 \mathrm{~m} / \mathrm{s}$ at $1 \mathrm{MHz}$ and density 1900 $\mathrm{kg} / \mathrm{m}^{3}$. Both shear and longitudinal waves were assumed to obey a power law in the middle layer (bone) with $y=0.96$ and $\alpha_{l}(\omega)=290 \mathrm{~Np} / \mathrm{m}$ and $\alpha_{s}(\omega)=590 \mathrm{~Np} / \mathrm{m}$ at $1 \mathrm{MHz}$. The choices of $\alpha_{1}$ and $\alpha_{s}$ were chosen so that $\alpha_{l}$ fell within the range of reported absorption coefficients at $1 \mathrm{MHz}$ and that the dispersive speeds of sound (shear and longitudinal) grew at similar rates. The top layer was assumed soft tissue, with speed of sound 1520 $\mathrm{m} / \mathrm{s}$ and density $1100 \mathrm{~kg} / \mathrm{m}^{3}$. The thickness of the solid layer was varied in the simulations. The photoacoustic signal was assumed to be recorded in a layer that is matched to the top layer a distance $d_{0}=1 \mathrm{~mm}$ away from the skin/bone edge. 

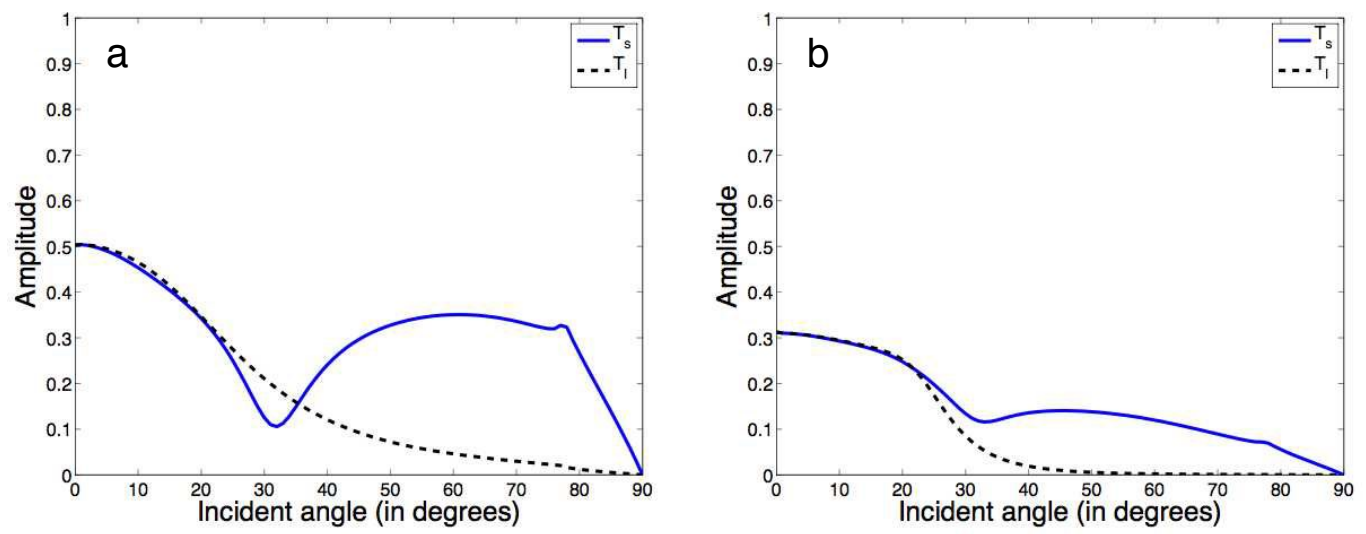

Figure 2. The magnitude of the ATFs, $\bar{T}_{l}(\theta, \omega)$ (dashed line) and $\bar{T}_{s}(\theta, \omega)$ (solid line), as a function of incident angle, for a bone layer with properties $d_{1}=2 \mathrm{~mm}$ and $\omega=2 \pi *(1.25 \mathrm{MHz})$ (panel a) and $\omega=2 \pi *(2.25 \mathrm{MHz})$ (panel b).

\section{TRANSFER FUNCTION ANALYSIS}

The possible errors that may be present by neglecting shear waves in the PAT image reconstruction algorithm are best understood by analyzing the ATF, $T\left(\mathbf{k}_{\|}, \omega\right)$, in Eq. (5), for the cases when shear waves are assumed to propagate in the solid layer and when shear waves are neglected. Let $\bar{T}_{s}(\theta, \omega)=T\left(\omega \sin (\theta) / c_{2}, \omega\right)$ denote the ATF for the layered medium when shear waves are modeled in layer 1. Let $\bar{T}_{l}(\theta, \omega)=T\left(\omega \sin (\theta) / c_{2}, \omega\right)$ denote the ATF for the layered medium when shear waves are not modeled in layer 1.

Plots of the magnitude of $\bar{T}_{s}$ and $\bar{T}_{l}$ as a function of angle are shown in Fig. 2 for two different temporal frequencies. In panel a, the assumed angular frequency is $\omega=2 \pi *(1.25 \mathrm{MHz})$ and in panel $\mathrm{b}, \omega=2 \pi *(2.25 \mathrm{MHz})$. In both panels, the thickness of the solid is assumed to be $2 \mathrm{~mm}$. One notes that the magnitude of $T_{l}$ and $T_{s}$ are nearly identical for angles less than $25^{\circ}$. Beyond that angle, the ATFs differ significantly. The critical angle for the fluid-solid interface at $1.25 \mathrm{MHz}$ is $\theta_{c}=\sin ^{-1} \frac{1483}{2957}=30.10^{\circ}$; that is, acoustic planewaves incident on the interface at an angle greater than $\theta_{c}$ correspond to non-propagating longitudinal waves in the solid layer (evanescent waves). Physically, for $\theta>\theta_{c}$, propagating shear waves are the primary means by which energy/information is transmitted across the solid layer. When shear waves are not modeled, there is no mechanism for these high spatial frequency waves to transmit from the layer containing the source (layer 2) to the layer in which detection occurs (layer 0). Accordingly, PAT reconstruction algorithms for planar, layered medium that do not account for the propagation of shear waves will yield images with a inferior/lower lateral spatial resolution than algorithms that account for shear waves.

Plots of the magnitude of $\bar{T}_{s}$ and $\bar{T}_{l}$ as a function of the thickness of the solid layer are shown in Fig. 3, with each panel representing a different angle of incidence. The assumed angular frequency is $\omega=2 \pi *(1.25 \mathrm{MHz})$. In panel $3 \mathrm{a}$, the incident planewave angle is $5^{\circ}$. One notes that $T_{s}$ and $T_{l}$ are similar for all thicknesses. In the limit that the thickness goes to zero, the magnitude of the ATF tends towards 0.9401, which coincides with the value of the transmission coefficient for normal incidence for a two-layer medium with properties consistent with layer 0 and layer 2. In panels b-d, the ATFs are shown for an incident angle of $20^{\circ}, 35^{\circ}$ and $50^{\circ}$, respectively. One sees that $T_{s}$ and $T_{l}$ differ as the incidence angle increases for any thickness of the bone, especially in panels $\mathrm{c}$ and $\mathrm{d}$ (corresponding to angles greater than the critical angle). For any incidence angle except zero degrees (normal incidence), the propagation of shear waves will have some effect on the transmission of acoustic energy from layer 2 to layer 0 , although that effect is more pronounced for larger angles.

To better understand the way in which not accounting for shear waves affects image fidelity, an error measure is introduced that is object-independent:

$$
E_{a}\left(\mathbf{k}_{\|}, \omega\right)=\frac{\left|T_{l}\left(\mathbf{k}_{\|}, \omega\right)\right|-\left|T_{s}\left(\mathbf{k}_{\|}, \omega\right)\right|}{\left|T_{s}\left(\mathbf{k}_{\|}, \omega\right)\right|} .
$$



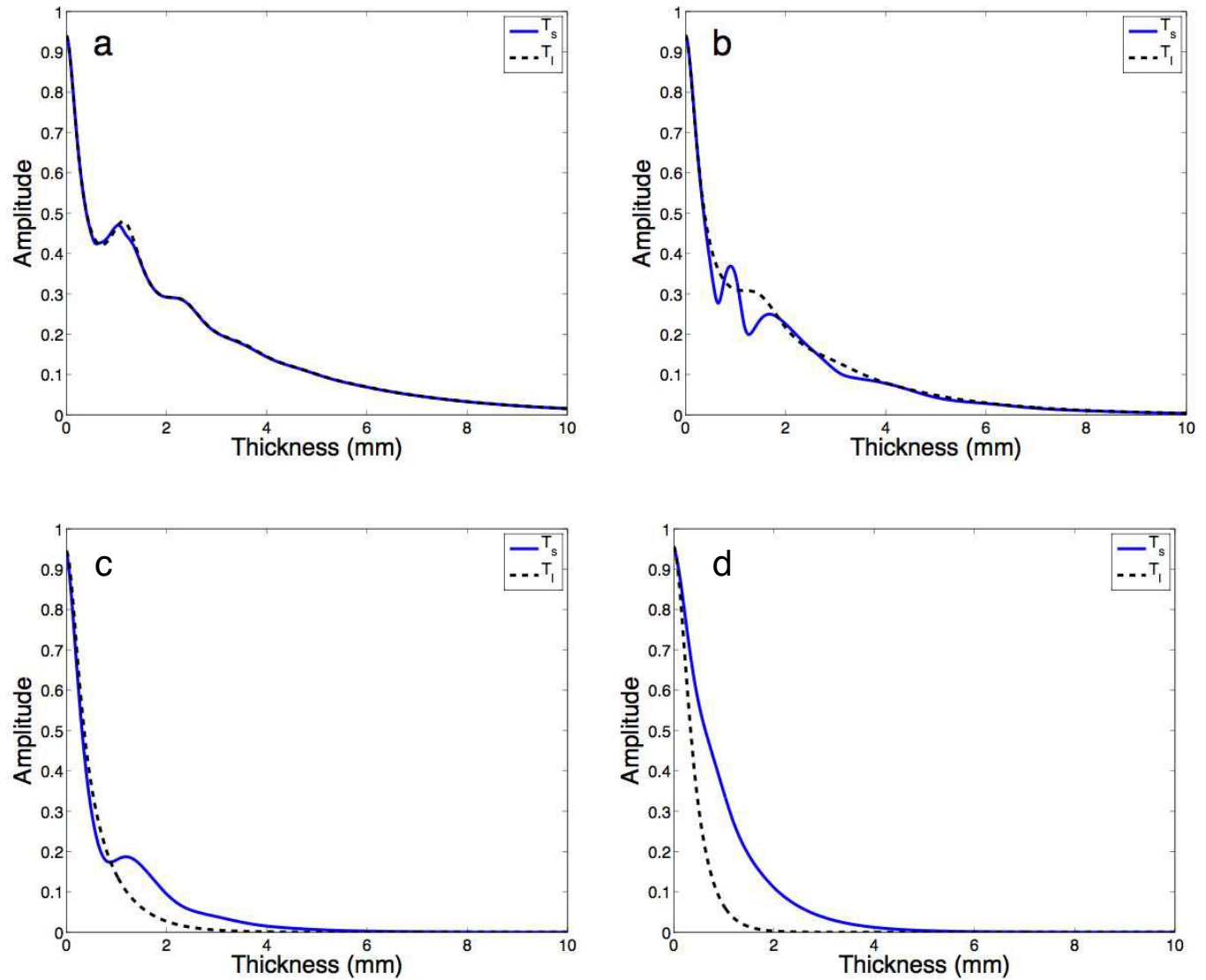

Figure 3. The magnitude of the ATFs, $\bar{T}_{l}(\theta, \omega)$ (dashed line) and $\bar{T}_{s}(\theta, \omega)$ (solid line), as a function of the thickness of the solid layer for planewaves at $\omega=2 \pi *\left(1.5 \mathrm{MHz}\right.$ ) at an incidence angle of $5^{\circ}$ (panel a), $20^{\circ}$ (panel b), 35 $($ panel c) and $50^{\circ}$ (panel d). 


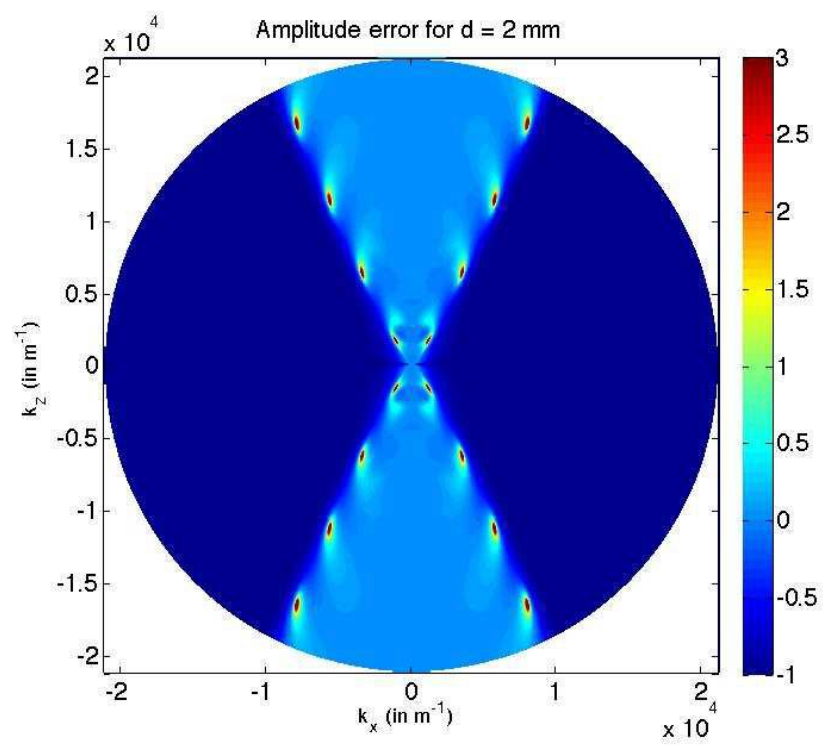

Figure 4. The amplitude error, $E_{a}$, inside the Ewald measurement sphere, for a solid with thickness $d=2 \mathrm{~mm}$. The maximum positive error in amplitude was set to be 3 to improve the dynamic range in the image.

$E_{a}$ denotes the estimated error in the amplitude of the Fourier component of the object. Note that $E_{a}$ can be both positive and negative. The positive error occurs when the model that omits shear waves overestimates the transmitted acoustic energy at a specific frequency and angle (see, for example, Fig. 2a from 25 to $35^{\circ}$ ). The negative error occurs when the model that omits shear waves underestimates the transmitted acoustic energy, with a maximum negative error of -1 when the ATF for the model that omits shear waves is zero.

In Fig. 4 , the amplitude error is shown in the $k_{x}-k_{z}$ plane for the case when the solid layer is $2 \mathrm{~mm}$ thick. It was assumed that the maximum measured frequency of the acoustic field in each case was $5 \mathrm{MHz}$, setting the radius of the Ewald measurement sphere at $k_{\max }=2 \pi 5 \mathrm{MHz} / c_{2}=21,184 \mathrm{~m}^{-1}$. Note that $E_{a}$ approaches -1 when $T_{l}$ is near zero, i.e. the model that does not account for shear waves predicts a null value for the transmission amplitude. One sees that the amplitude error is near -1 for all incidence angles above $\theta_{c}$. This severely limits the ability of a non-shear wave based method for resolving large transverse frequency components of the to-be-imaged object in a planar detection geometry. In this figure, the maximum positive error in amplitude was set to be 3 . The large positive error locations correspond to points at which $T_{s}$ approaches 0 .

One notes that these findings generalize beyond specific system parameters used in this paper. Varying the values of $\alpha_{m}$ and $y$ will affect the rate at which the ATFs fall off as a function of thickness in Fig. 3; however, the relationship between $T_{s}$ and $T_{l}$ will remain similar. Likewise, using different values for the speeds of sound or densities in the solid and fluid layers will change the critical angle, $\theta_{c}$, but not the fact that $T_{s}$ and $T_{l}$ are in good agreement when the incident angle is smaller than the critical angle.

\section{SUMMARY AND FUTURE WORK}

In this work, a recently-introduced PAT algorithm was employed to determine the role shear waves play in PAT image formation. Computer-simulations demonstrated that omitting shear waves from the imaging model results in a smaller number of spatial frequency components of the object being estimable than when shear waves are included. The spatial frequency components that are not accurately estimated correspond to Fourier components parallel or near-parallel to the layer interfaces and are consistent with planewave components incident beyond the critical angle of the solid-fluid interface. 
The electrical impulse response, due to transduction, and the spatial impulse response, due to the finite size and number of the transducers, can be modeled as filters. ${ }^{20,28}$ These effects can be incorporated into the imaging model presented in this work by including a multiplicative factor in the denominator of the right hand side of Eq. (5). One notes that the electrical impulse response often serves to diminish very low temporal frequency components of the data. Depending on certain geometrical factors, the spatial pass-band of the transducer may diminish data from Ewald's sphere that correspond to high transverse spatial frequencies. These data correspond to the cases for which the largest errors occur by not accounting for shear waves in the reconstruction algorithm. Much wider pass-bands in both spatial and temporal frequencies occur when using planar, polymerfilm ultrasound sensors ${ }^{29}$ to acquire the photoacoustic signal.

There are a number of open problems that remain to be addressed in regards to accounting for shear wave physics in transcranial PAT. The manner in which neglecting shear waves affects image quality when different image reconstruction algorithms and non-planar imaging geometries are employed has not been investigated systematically. The interplay between transducer placement/geometry and the errors described in this manuscript associated with neglecting shear waves is also an important topic of future investigation. When the shear wave properties are not known, it may be possible to develop an iterative reconstruction algorithm which only uses the data consistent with planewaves that are incident at an angle below the critical angle in the reconstruction. The development of such an algorithm remains a topic for future work.

\section{Acknowledgments}

Robert W. Schoonover and Mark A. Anastasio acknowledge support in part by NIH awards EB010049 and EB009715. Lihong V. Wang acknowledge support by the NIH awards EB010049, CA134539, EB000712, CA136398, and EB008085. L.V.W. has a financial interest in Microphotoacoustics, Inc. and Endra, Inc., which, however, did not support this work.

\section{REFERENCES}

1. L. V. Wang, "Prospects of photoacoustic tomography," Med. Phys. 35, 5758 (2008).

2. M. Xu and L. V. Wang, "Biomedical photoacoustics," Rev. Sci. Instrum. 77, 041101 (2006).

3. A. A. Oraevsky and A. A. Karabutov, "Optoacoustic tomography," in "Biomedical Photonics Handbook," , T. Vo-Dinh, ed. (CRC Press LLC, 2003).

4. L. Wang, ed., Photoacoustic Imaging and Spectroscopy (CRC, 2009).

5. X. Wang, Y. Pang, G. Ku, X. Xie, G. Stoica, and L. Wang, "Noninvasive laser-induced photoacoustic tomography for structural and functional in vivo imaging of the brain," Nat. Biotechnol. 21, 803-806 (2003).

6. S. Yang, Q. Zhou, L. Xiang, and Y. Lao, "Functional imaging of cerebrovascular activities in small animals using high-resolution photoacoustic tomography," Med. Phys. 34, 3294 (2007).

7. H. Brecht, R. Su, M. Fronheiser, S. Ermilov, A. Conjusteau, and A. Oraevsky, "Whole-body threedimensional optoacoustic tomography system for small animals," J. Biomed. Opt. 14, 064007 (2009).

8. V. Ntziachristos and D. Razansky, "Molecular imaging by means of multispectral optoacoustic tomography (MSOT)," Chem. Rev. 110, 2783-2794 (2010).

9. R. Kruger, D. Reinecke, and G. Kruger, "Thermoacoustic computed tomography- technical considerations," Med. Phys. 26, 1832-1837 (1999).

10. M. Haltmeier, O. Scherzer, P. Burgholzer, and G. Paltauf, "Thermoacoustic computed tomography with large planar receivers," Inverse Probl. 20, 1663-1673 (2004).

11. P. Ephrat, L. Keenliside, A. Seabrook, F. S. Prato, and J. J. L. Carson, "Three-dimensional photoacoustic imaging by sparse-array detection and iterative image reconstruction," J. Biomed. Opt. 13, 054052 (2008).

12. B. T. Cox, S. R. Arridge, K. P. Kstli, and P. C. Beard, "Two-dimensional quantitative photoacoustic image reconstruction of absorption distributions in scattering media by use of a simple iterative method," Appl. Opt. 45, 1866-1875 (2006). 
13. K. Wang, S. Ermilov, R. Su, H. Brecht, A. Oraevsky, and M. Anastasio, "An Imaging Model Incorporating Ultrasonic Transducer Properties for Three-Dimensional Optoacoustic Tomography." IEEE T. Med. Imaging (In press, 2010).

14. Y. Xu and L. Wang, "Rhesus monkey brain imaging through intact skull with thermoacoustic tomography," IEEE T. Ultrason. Ferr. 53, 542-548 (2006).

15. X. Jin, C. Li, and L. V. Wang, "Effects of acoustic heterogeneities on transcranial brain imaging with microwave-induced thermoacoustic tomography," Med. Phys. 35, 3205 (2008).

16. X. Yang and L. V. Wang, "Monkey brain cortex imaging by photoacoustic tomography," J. Biomed. Opt. 13, 044009 (2008).

17. L. Nie, Z. Guo, and L. Wang, "Photoacoustic tomography of monkey brain using virtual point ultrasonic transducers," J. Biomed. Opt. 16, 076005 (2011).

18. B. E. Treeby, E. Z. Zhang, and B. T. Cox, "Photoacoustic tomography in absorbing acoustic media using time reversal," Inverse Probl. 26, 115003 (2010).

19. R. W. Schoonover and M. A. Anastasio, "Compensation of shear waves in photoacoustic tomography with layered acoustic media," J. Opt. Soc. Am. A 28, 2091-2099 (2011).

20. Y. Xu, D. Feng, and L. V. Wang, "Exact frequency-domain reconstruction for thermoacoustic tomography: I. Planar geometry," IEEE T. Med. Imaging 21, 823-828 (2002).

21. R. W. Schoonover and M. A. Anastasio, "Image reconstruction in photoacoustic tomography involving layered acoustic media," J. Opt. Soc. Am. A 28, 1114-1120 (2011).

22. P. Droin, G. Berger, and P. Laugier, "Velocity dispersion of acoustic waves in cancellous bone," IEEE T. Ultrason. Ferr. 45, 581-592 (1998).

23. K. Waters, M. Hughes, J. Mobley, G. Brandenburger, and J. Miller, "On the applicability of KramersKrönig relations for ultrasonic attenuation obeying a frequency power law," J. Acoust. Soc. Am. 108, 556 (2000).

24. P. White, G. Clement, and K. Hynynen, "Local frequency dependence in transcranial ultrasound transmission," Phys. Med. Biol. 51, 2293 (2006).

25. K. Waters and B. Hoffmeister, "Kramers-Kronig analysis of attenuation and dispersion in trabecular bone," J. Acoust. Soc. Am. 118, 3912 (2005).

26. S. Chaffaï, F. Padilla, G. Berger, and P. Laugier, "In vitro measurement of the frequency-dependent attenuation in cancellous bone between 0.2 and 2 mhz," J. Acoust. Soc. Am. 108, 1281 (2000).

27. S. Pichardo, V. Sin, and K. Hynynen, "Multi-frequency characterization of the speed of sound and attenuation coefficient for longitudinal transmission of freshly excised human skulls," Phys. Med. Biol. 56, 219 (2011).

28. K. Wang, S. Ermilov, R. Su, H. Brecht, A. Oraevsky, and M. Anastasio, "An imaging model incorporating ultrasonic transducer properties for three-dimensional optoacoustic tomography," IEEE T. Med. Imaging. 30, 203-214 (2011).

29. E. Zhang, J. Laufer, and P. Beard, "Backward-mode multiwavelength photoacoustic scanner using a planar fabry-perot polymer film ultrasound sensor for high-resolution three-dimensional imaging of biological tissues," Appl. Opt. 47, 561-577 (2008).

30. R. Schulze, G. Zangerl, D. Meyer, F. Handle, R. Nuster, G. Paltauf, and O. Scherzer, "On the use of frequency-domain reconstruction algorithms for photoacoustic imaging," J. Biomed. Opt. 16, 086002 (2011). 